\title{
Cincuenta casos de metabolismo basal en el embarazo a término
}

\author{
POR LeOPOLdo SAAVEdRa
}

Presentamos a continuación en un cuadro-resumen las historias y los resultados del examen del metabolismo basal practicado a cincuenta mujeres embarazadas a término, de los distintos servicios del Instituto Materno-Infantil "Concepción Villaveces de Acosta".

Las pacientes escogidas presentaban embarazos clínicamente normales y el examen objeto de nuestro estudio lo hicimos con un aparato eléctrico portátil que nos ofreció la ventaja de tomar las gráficas a la cabecera de la gestante. También nos valimos cel Laboratorio de Metabolismo Basal del Hospital de San Juan de Dios, por gentil autorización del Profesor José del Carmen Acosta, a quien expresamos nuestro agradecimiento.

Las gráficas tomadas en dicho laboratorio se hicieron en un aparato "Sanborn" de mecanismo semejante al usado por nosotros en el Instituto.

Los aparatos constan de un mecanismo formado por un espirómetro, constituído por un fuelle, un tambor inscriptor movido por un aparato eléctrico de relojería. Por ser del sistema de circuito cerrado, el gas usado es el oxígeno y para la absorción, del C 02 se usa soda o potasa.

No entramos en detalles en la descripción de los aparatos, pues no es éste el objeto de nuestro estudio. Tan solo anotamos que el perfeccionamiento de estos instrumentos facilita en gran forma el examen y la interpretación de las gráficas; el cálculo de la cifra que representa el metabolismo, se obtiene en forma sencilla por medio de una regla parecida a la que usan los ingenieros. 


\section{TECNICA}

La persona examinada permaneció en ayuno desde doce a catorce horas antes. Ultima comida del día anterior poco abundante; el metabolismo se tomó entre las ocho y las nueve de la mañana, estando la persona acostada lo más cómodamente posible, en equilibrio térmico, en prefecto reposo muscular y mental. La noche anterior se le toma la talla y el peso advirtiéndole en forma cordial que al día siguiente por la mañana se le va a practicar un examen sencillo que no le proporcionará ninguna molestia y que por el contrario, redundará en bien de su salud. Al día siguiente, antes de la prueba, se le hace un examen clínico y se insiste en inspirarle confianza para que colabore, lo cual es de mucha importancia. Se le enseña luego a respirar por la boca ciurante algunos minutos aplicándole una pinza especial en la nariz para obturar los conductos nasales; después se le hace respirar con la boquilla de caucho. Una vez que está respirando tranquila, se conecta el aparato a la boquilla y se introduce el oxígeno a la cámara. Antes de poner a funcionar el sistema eléctrico es conveniente hacer respirar a la paciente, unas cinco o seis veces antes de empezar a marcar la gráfica. Durante toda la inscripción de la gráfica se vigila ésta, y al considerarse terminada se retira la aguja inscriptora y luego la placa. Se hace el cálculo del oxígeno consumido en un minuto y si la gráfica es satisfactoria se da por terminada la prueba.

Con las precauciones anotadas en forma somera, las gráficas obtenidas fueron todas satisfactorias y los resultados que transcribimos en la última columna de nuestro cuadro resumen fueron conseguidos en esta forma.

A continuación presentamos en un cuadro, el resumen de las historias de las pacientes examinadas. Anotamos en una columna especial, el estado del tiroides limitándonos a observar si había o no hipertrofia de dicha glándula.

Haremos algunos comentarios sobre esta observación concediéndole mucha importancia, por ser de tanta trascendencia su papel en el metabolismo basal del individuo. 
METABOLISMO BASAL EN CINCUENTA EMBARAZADAS A TERMINO

\begin{tabular}{|c|c|c|c|c|c|c|c|c|c|c|c|c|c|}
\hline \multirow[b]{2}{*}{ Caso } & \multirow[b]{2}{*}{ Hist. } & \multirow[b]{2}{*}{ Edad } & \multirow[b]{2}{*}{ Paridad } & \multirow[b]{2}{*}{ Peso } & \multirow[b]{2}{*}{ Talla } & & \multirow[b]{2}{*}{ Tiemp. } & \multicolumn{2}{|c|}{ TIROIDES } & \multicolumn{2}{|c|}{ EMBARAZO } & \multirow[b]{2}{*}{ Met. B. } \\
\hline & & & & & & Pulso & T. A. & & Hip. & Norm. & Norm. & Pat. & \\
\hline 1 & 6304 & 20 & 2 & 158 & $601 / 4$ & 76 & $10 \times 7$ & 36.4 & & $\mathrm{x}$ & $\mathrm{x}$ & & $+13 \%$ \\
\hline 2 & 6563 & 29 & 5 & 136 & $601 / 4$ & 65 & $10 \times 6$ & 36.2 & & $\mathrm{x}$ & $\mathrm{x}$ & & $+19 \%$ \\
\hline 3 & 5592 & 22 & 2 & 145 & $653 / 4$ & 80 & $8.5 \times 6$ & 36.8 & & $x$ & $\mathrm{x}$ & & $+\quad 8 \%$ \\
\hline 4 & 6813 & 22 & 1 & 144 & $603 / 4$ & 78 & $8 \times 5$ & 36.3 & $\mathrm{x}$ & & $\mathrm{x}$ & & $+18 \%$ \\
\hline 5 & 5181 & 17 & 1 & 125 & $571 / 2$ & 73 & $11 \times 7$ & 36.4 & & $\mathrm{x}$ & $\mathrm{x}$ & & $+22 \%$ \\
\hline 6 & 6563 & 29 & 5 & 136 & $601 / 4$ & 65 & $10 \times 6$ & 36.3 & $\mathrm{x}$ & & $\mathrm{x}$ & & $+19 \%$ \\
\hline 7 & 6518 & 20 & 1 & 140 & 59 & 70 & $11 \times 6$ & 36.2 & & $\mathrm{x}$ & $\mathrm{x}$ & & $+50 \%$ \\
\hline 8 & 5989 & 19 & 2 & 145 & 57 & 80 & $9.6 \times 5$ & 36.6 & & $x$ & $\mathrm{x}$ & & $+25 \%$ \\
\hline 9 & 6090 & 20 & 1 & 100 & 57 & 75 & $7.5 \times 4$ & 36.6 & $\mathrm{x}$ & & $x$ & & $+\quad 5 \%$ \\
\hline 10 & 6331 & 35 & 8 & 135 & $571 / 4$ & 78 & $12 \times 7$ & 36.5 & & $\mathrm{x}$ & & & $+35 \%$ \\
\hline 11 & 6381 & 29 & 8 & 128 & $583 / 4$ & 80 & $12 \times 7$ & 36.6 & $\mathrm{x}$ & & & & $+19 \%$ \\
\hline 12 & 6414 & 21 & 3 & 104 & 54 & 76 & $9 \times 6$ & 36.6 & & $\mathrm{x}$ & $x$ & & $+28 \%$ \\
\hline 13 & 6410 & 36 & 6 & 130 & 54 & 80 & $10 \times 7$ & 36.2 & & $x$ & $x$ & & $\begin{array}{r}+5 \% \\
+\quad 5 \%\end{array}$ \\
\hline 14 & 4972 & 28 & 2 & 169 & 63 & 62 & $13 \times 9$ & 36.2 & $\mathrm{x}$ & & $\mathrm{x}$ & & $+8 \%$ \\
\hline 15 & 6380 & 19 & 1 & 106 & $571 / 4$ & 75 & $10 \times 5$ & 36.5 & $\mathrm{x}$ & & $\mathrm{x}$ & & $+26 \%$ \\
\hline 16 & 5965 & 29 & 3 & 104 & $553 / 4$ & 78 & $9 \times 5$ & 36.4 & $\mathrm{x}$ & & $\mathrm{x}$ & & $+6 \%$ \\
\hline 17 & 6636 & 25 & 5 & 119 & $561 / 2$ & 60 & $12 \times 7$ & 36.5 & $x$ & & $x$ & & $+70 \%$ \\
\hline 18 & 7032 & 24 & 1 & 136 & $561 / 2$ & 70 & $9.5 \times 6$ & 36.4 & $x$ & & $x$ & & $+5 \%$ \\
\hline 19 & 7152 & 19 & 1 & 119 & 63 & 74 & $12 \times 8$ & 36.5 & $\mathrm{x}$ & & $\mathrm{x}$ & & $+20 \%$ \\
\hline 20 & 5406 & 27 & 2 & 126 & 60 & 78 & $10 \times 6$ & 36.4 & & $\mathrm{x}$ & $\mathrm{x}$ & & $+16 \%$ \\
\hline 21 & 6655 & 19 & 2 & 127 & 59 & 88 & $11 \times 7$ & 36.4 & $\mathrm{x}$ & & $\mathrm{x}$ & & $+5 \%$ \\
\hline 22 & 6683 & 17 & 1 & 129 & 61 & 75 & $10 \times 6$ & 36.2 & $\mathrm{x}$ & & $x$ & & $-18 \%$ \\
\hline 23 & 6399 & 18 & 1 & 140 & 60 & 68 & $9 \times 5$ & 36.2 & $\mathrm{x}$ & & $\mathrm{x}$ & & $8 \%$ \\
\hline 24 & 7297 & 19 & 1 & 127 & 59 & 60 & $10 \times 6$ & 36.5 & $\mathrm{x}$ & & $\mathrm{x}$ & & $-3 \%$ \\
\hline 25 & 6821 & 18 & 1 & 145 & 59 & 60 & $8 \times 4$ & 36.4 & & $\mathrm{x}$ & $x$ & & $+\quad 1 \%$ \\
\hline
\end{tabular}




\section{METABOLISMO BASAL EN CINCUENTA EMBARAZADAS A TERMINO}

\begin{tabular}{|c|c|c|c|c|c|c|c|c|c|c|c|c|c|}
\hline \multirow{2}{*}{ Caso } & \multirow[b]{2}{*}{ Hist. } & \multirow[b]{2}{*}{ Edad } & \multirow[b]{2}{*}{ Paridad } & \multirow[b]{2}{*}{ Peso } & \multirow[b]{2}{*}{ Talla } & \multirow[b]{2}{*}{ Pulso } & \multirow[b]{2}{*}{ T. A. } & \multirow[b]{2}{*}{ Tiemp. } & \multicolumn{2}{|c|}{ TIROIDES } & \multicolumn{2}{|c|}{ EMBARAZO } & \multirow[b]{2}{*}{ Met. B. } \\
\hline & & & & & & & & & Hip. & Norm. & Narm. & Pat. & \\
\hline 26 & 6499 & 21 & 1 & 125 & 62 & 80 & $9 \times 5$ & 36.5 & & $\mathrm{x}$ & $\mathrm{x}$ & & $+5 \%$ \\
\hline 27 & 6813 & 18 & 1 & 116 & 57 & 65 & $9 \times 6$ & 36.8 & & $x$ & $\mathrm{x}$ & & $4 \%$ \\
\hline 28 & 7105 & 21 & 1 & 121 & 60 & 55 & $10 \times 5$ & 37 & $\mathrm{x}$ & & $\mathrm{x}$ & & $8 \%$ \\
\hline 29 & 6824 & 23 & 2 & 123 & 58 & 63 & $10 \times 5$ & 36.5 & & $\mathrm{x}$ & $\mathrm{x}$ & & $0 \%$ \\
\hline 30 & 6339 & 20 & 2 & 151 & 61 & 89 & $8.5 \times 5$ & 36.1 & $\mathrm{x}$ & & $\mathrm{x}$ & & $5 \%$ \\
\hline 31 & 6261 & 20 & 1 & 121 & 58 & 65 & $10 \times 7$ & 36.1 & & $\mathrm{x}$ & $\mathrm{x}$ & & $5 \%$ \\
\hline 32 & 7079 & 22 & 1 & 138 & 61 & 65 & $12 \times 7$ & 37 & $x$ & & $\mathrm{x}$ & & $+5 \%$ \\
\hline 33 & 6990 & 18 & 1 & 111 & 59 & 65 & $12 x^{\prime} 7$ & 37 & & $\mathrm{x}$ & $x$ & & $-13 \%$ \\
\hline 34 & 6874 & 35 & 4 & 147 & $601 / 2$ & 90 & $11 x 5$ & 37.1 & $\mathrm{x}$ & & $\mathrm{x}$ & & $+32 \%$ \\
\hline 35 & 6321 & 18 & 1 & 127 & 61 & 82 & $10 \times 7$ & 36.4 & & $\mathrm{x}$ & $x$ & & $+8 \%$ \\
\hline 36 & 6932 & 25 & 1 & 114 & $583 / 4$ & 80 & $9 \times 5$ & 36.3 & & $x$ & $\mathrm{x}$ & & $+27 \%$ \\
\hline 37 & 1100 & 22 & 4 & 136 & 60 & 67 & $12 \times 7$ & 36.8 & & $\mathrm{x}$ & $\mathrm{x}$ & & $+19 \%$ \\
\hline 38 & 0381 & 42 & 2 & 112 & 58.5 & 78 & $9 \times 6$ & 36.7 & & $\mathrm{x}$ & $x$ & & $+3 \%$ \\
\hline 39 & 1113 & 34 & 5 & 118 & 60 & 85 & $11 x 8$ & 36.8 & & $x$ & $x$ & & $1 \%$ \\
\hline 40 & 6901 & 25 & 2 & 147 & 61 & 60 & $9 \times 5$ & 36.5 & & $x$ & $\mathrm{x}$ & & $0 \%$ \\
\hline 41 & 7358 & 28 & 1 & 123 & 60 & 65 & $10 \times 7$ & 36.5 & $\mathrm{x}$ & & $\mathrm{x}$ & & $+8 \%$ \\
\hline 42 & 0304 & 26 & 2 & 121 & 55 & 60 & $10 \times 7$ & 36.3 & $\mathrm{x}$ & & $x$ & & $+14 \%$ \\
\hline 43 & 0180 & 25 & 2 & 151 & 64 & 68 & $11 \times 7$ & 36.5 & & $x$ & $\mathrm{x}$ & & $-11 \%$ \\
\hline 44 & 7339 & 23 & 1 & 125 & 59 & 70 & $10 \times 6$ & 36.8 & & $\mathrm{x}$ & $\mathrm{x}$ & & $0 \%$ \\
\hline 45 & 0174 & 15 & 1 & 123 & 59 & 65 & $10 \times 7$ & 37 & $\mathrm{x}$ & & $\mathrm{x}$ & & $-17 \%$ \\
\hline 46 & 2533 & 38 & 2 & 129 & 57 & 72 & $11 \times 6$ & 36.4 & & $x$ & $\mathrm{x}$ & & $+6 \%$ \\
\hline 47 & 2660 & 26 & 7 & 127 & 56.5 & 68 & $12 \times 7$ & 36.6 & & $\mathrm{x}$ & $\mathrm{x}$ & & $+24 \%$ \\
\hline 48 & 2662 & 40 & 5 & 110 & 56 & 68 & $13 x 8$ & 37 & & $\mathrm{x}$ & $\mathrm{x}$ & & $+8 \%$ \\
\hline 49 & 0505 & 23 & 2 & 138 & 62 & 72 & $11 \times 6$ & 36.5 & $\mathrm{x}$ & & $\mathrm{x}$ & & $+16 \%$ \\
\hline 50 & 0347 & 22 & 2 & 140 & 61 & 95 & $10 \times 6$ & 36.8 & $x$ & & $\mathrm{x}$ & & $+27 \%$ \\
\hline
\end{tabular}




\section{METABOLISMO EN EL EMBARAZO}

Diferentes autores, entre elios Magnus Levy, encontraron un aumento de un $17 \%$ en la absorción de oxígeno en las embarazadas. Hanna en 1938, junto con otros investigadores, encuentran que la gestante normal a término el metabolismo basal está reguiarmente aumentado en cifras que oscilan entre más 20 y más $30 \%$ como término medio. Anotan también que algunas embarazadas normales presentan un aumento hasta del $70 \%$.

En nuestras observaciones pudimos comprobar un caso en que el metabolismo basal era de más $50 \%$ en un embarazo normal y otro caso en que era de más $70 \%$ también normal. Así mismo se vio que hubo 4 casos en que el M. B. se encontró entre menos 10 y menos $20 \%$ en embarazos también normales.

Bayer al estudiar ei M. B. en 44 embarazadas a término normales encontró un aumento de más 33 a más 35 por ciento en comparación con mujeres no embarazadas de la misma superficie corporal.

Sadiford y Weler observaron el M. B. antes, durante y seis meses después del parto y dedujeron que durante el último período del embarazo el M. B. aumenta en un promedio de más 25 por ciento. No encontraron aumento durante el puerperio y la lactancia.

Peralta Ramos, de La Argentina, sobre um total de 70 embarazadas encontró los siguientes resultados: en el 61\% los valores están por encima de lo normal; en el $33 \%$ dentro de lo normal y en seis por ciento, disminuidos.

También comprobó que el aumento se realiza en el último pelíodo dei' embarazo. El aumento observado por este autor fue en la siguiente proporción por orden de frecuencias:

$$
\begin{aligned}
& \text { De }+11 \% \text { a }+20 \% \text { en } 25 \text { casos. } \\
& \text { De }+20 \% \text { a }+30 \% \text { en } 11 \text { casos. } \\
& \text { De }+30 \% \text { a }+40 \% \text { en } 7 \text { casos. }
\end{aligned}
$$

En 4 casos observó disminución hasta de $-19 \%$.

En 10 casos comprobó que el M. B. permaneció dentro de límites normales.

Ei doctor Gonzalo Duque Gómez en su Tesis de Grado en 1947 observó un aumento en la siguiente proporción sobre un totai de 8 casos: 


$$
\begin{aligned}
& \text { De }+11 \% \text { a }+20 \% \text {, en } 24 \text { casos. } \\
& \text { De }+21 \% \text { a }+30 \% \text {, en } 33 \text { casos. } \\
& \mathrm{De}^{\prime}+31 \% \mathrm{a}+40 \% \text {, en } 8 \text { casos. } \\
& \text { De }+41 \% \text { a }+50 \% \text {, en } 4 \text { casos. } \\
& \mathrm{De}+73 \% \text { en } 1 \text { caso. }
\end{aligned}
$$

Total de casos de metabolismo basal aumentado: 70. Los 14 casos restantes permanecieron dentro de límites normales.

Como se puede apreciar fácilmente, la mayor frecuencia correspondió a una cifra que oscila entre $+20 \mathrm{y}+30 \%$.

En nuestro trabajo hemos observado 50 casos de embarazos a término normales, de los cuales presentamos ei siguiente cuadro de frecuencias:

$$
\begin{aligned}
& \text { De - } 10 \% \text { a }-20 \% 5 \text { casos. } \\
& \text { De } \quad 0 \% \text { a - } 9 \% \quad 8 \text { casos. } \\
& \mathrm{De}+1 \% \mathrm{a}+9 \% 13 \text { casos. } \\
& \text { De }+10 \% \mathrm{a}+19 \% 11 \text { casos. } \\
& \mathrm{De}+20 \% \mathrm{a}+29 \% \text { casos. } \\
& \text { De }+30 \% \mathrm{a}+39 \% 2 \text { casos. } \\
& \mathrm{De}+50 \% \quad 1 \text { caso. } \\
& \text { De }+70 \% \quad 1 \text { caso. }
\end{aligned}
$$

Total de casos de M. B. aumentado: 24. Que corresponden a un $48 \%$.

Casos que permanecen dentro de límites normales: 21: Que corresponden a un $42 \%$.

Casos de M. B. disminuído: 5. Que corresponden a un $10 \%$.

Observando nuestro cuadro de frecuencias, deducimos que el mayor número de casos está comprendido entre las cifras de $+1 \%$ $\mathrm{y}+10 \%$. Es decir, 13 casos de los cincuenta observados tienen cifras metabólicas entre $+1 \mathrm{y}+10 \%$.

Hay que anotar sinembargo que el número de casos entre $+10 \mathrm{y}+20 \%$ es sensiblemente igual.

De esto podemos concluir que el metabolismo del embarazo normal a término está comprendido en cifras entre $+1 \% \mathrm{y}+$ $20 \%$ en los casos observados en pacientes hospitalizadas en los Servicios de Caridad.

\section{GLANDULAS ENDOCRINAS Y EMBARAZO}

La complejidad de sus funciones expilica que estos órganos experimenten modificaciones importantes durante el embarazo. 


\section{Tiroides}

Desde los tiempos de Hipócrates ya fue anotado el aumento de tamaño que experimenta esta glándula en la gestación.

La hipertrofia tiroidea gravídica se considera debida a un aumento de secreción y acumulación de éste en la cavidad de las ve-sículas. Según varios autores, entre ellos Pende, en vez de existir hiperfunción del tiroides, lo que existe es disminución de la excreción coioidea.

Debe anotarse que aun cuando falte el tiroides, es posible la gestación; si bien puede interrumpirse por esta causá, también puede llegar a término. La insuficiencia tiroidea sueie traer como consecuencia hipogénesis mamaria y disminución de la secreción glandular. Se ha comprobado que si la función tiroidea es normal, la secreción láctea es abundante.

Se sabe también que el embarazo existe un hipertiroidismo más o menos marcado, pero en casos normales compensado. Se ha encontrado en la sangre de la gestante una substancia, hormona tiroidea, que eleva el metabolismo basal y la cetonemia y rebaja el glucógeno hepático. Pero en ia sangre de la embarazada también circula hormona tirotropa, lo cual contribuye así mismo a la estimulación del tiroides e indirectamente al aumento de las oxidaciones y por consiguiente al aumento del metabolismo basal.

\section{Hipófisis.}

El descubrimiento de las hormonas hipofisarias ha motivado determinados estudios de su constitución anatómica especialmente del lóbulo anterior que se manifiesta hipertrofiado en el estado gravídico.

Esta hipertrofia del lóbuio anterior parece que alcanza a todos sus elementos. Para algunos autores, depende solamente de la formación de células especiales por hipertrofia e hiperplasia de las células fundamentales o cromófobas, sin que aumenten las acidófilas y basófiias. Estas empiezan a disminuír al terminar el embarazo y al final del puerperio son nuevamente células ordinarias. Según experiencias en animales hipofisectomizados, la mortalidad en perras gestantes es del 100\%, mientras solo es del $66 \%$ en los mismos animales fuera del estado de gravidez. 


\section{Hormonas hipofisiarias.}

Las hormonas metabólicas dei lóbulo anterior que actúan sobre el metabolismo hidrocarbonado, sobre la fase desasimilativa son las siguientes:

La hormona contrainsular de Lucke, la hormona diabetógena de Houssay, la hormona glucocogenolitica de Anselmino y Hoffmann. El estado actual de ias investigaciones a este respecto, hace creer que estas hormonas se encuentran aumentadas en ia sangre de las embarazadas y que la desglucogenización hepática, podría explicarse por tal mecanismo.

Factores activos sobre el metabolismo graso se han descrito dos: la lipotrina de Raab (1931), que este autor encuentra en la sangre de las embarazadas y la substancia acetonemiante de Anselmino y Hoffmann (1934), y a ias cuales sus descubridores atribuyen la acidosis de la hiperemesis gravídica.

Las hormonas del lóbulo posterior que influyen sobre la presión arterial y sobre el metabolismo del agua en las gestantes, según Anselmino y Hoffmann, son la antidiurética y la vasopresina. Levy-Solal (1935), creen que estas hormonas son el factor decisivo en la génesis de los edemas gravídicos.

\section{Paratiroides.}

Se cree actualmente, que a pesar de que la movilización del calcio en la embarazada aumenta considerablemente, lo mismo que su consumo y absorción fetal, el no presentar la embarazada accesos de tetania, se debe a una hiperproducción de paratiroidina, cuya circulación está aumentada en la gestación según lo han demostrado Hoffmann y Rhoden (1933).

\section{Suprarrenales.}

Se ha sostenido que existe durante ei embarazo un estado relativo de insuficiencia corticorrenal (Rimi 1939). Según recientes investigaciones, se ha comprobado que más bien hay una hiperfunción de esta glándula, durante la gestación, pero que debido a las grandes necesidades del organismo de estas hormonas, la corteza suprarrenal no alcanza el rendimiento exigido y el organismo da síntomas de insuficiencia. Anotamos que el descen- 
so de la tensión arterial que se encuentra con tanta frecuencia en las embarazadas, puede obedecer a esta causa.

En ia medula de las suprarrenales, son numerosas las observaciones que señalan un aumento del tejido cromafin. La adrenalina se comporta además como antagonista de la insulina, la cual, como se sabe, controia el estado de la glicemia; esto nos podría explicar en parte, la hipoglicemia que se observa con frecuencia en el embarazo, a pesar de haber un aumento en el metaboiismo de los hidrocarbonados.

\section{Pancreas.}

Varias investigaciones han puesto de presente un moderado hiperinsulinismo durante el embarazo. Esto nos explicaría en parte las mejorías pasajeras de la diabetes en las embarazadas.

En cuanto a la acción de la placenta sobre el metabolismo de la embarazada, no está bien establecida; pero se acepta que sus hormonas actúan indirectamente sobre el metabolismo, obrando sobre las demás giándulas endocrinas.

\section{CAUSAS DEL AUMENTO DEL METABOLISMO BASAL EN EL EMBARAZO}

Como hemos podido observar por los trabajos realizados por diferentes autores citados, y por nuestras comprobaciones en cincuenta casos de embarazadas a término, el metabolismo basal en las gestantes está aumentado. Este aumento ha sido interpretado en diversas formas y no hay un acuerdo completo en las causas que lo determinan. Para algunos, es el producto de los procesos oxidativos del embrión superpuesto a los de la madre. Para otros de una acción por parte de todo ei organismo de la madre.

Hay un hecho evidente que es la hiperfunción del tiroides en ei embarazo que nos podría explicar este aumento.

En cuanto a los procesos fisiológicos que más influyen en las oxidaciones metabólicas como son, la respiración y la circulación, sufren importantes cambios que luego expondremos en forma somera.

La respiración se modifica, aumentando su frecuencia y la movilización del aire de reserva. Se produce así una hiperventiación, la cual influye sobre la sangre produciendo una alcalosis gaseosa, la cual a su vez obra sobre los centros resp.ratorios. 
Los cambios circulatorios principales se refieren al aumento del volumen-minuto circuiatorio (Montalvo. 1944).

Resumen del metabolismo de Glúcidos, Prótidos, Lípidos sales minerales principales y agua y sus modificaciones en el embarazo.

\section{Glúcidos.}

La ingestión de hidratos de carbono generaimente aumenta considerablemente en las embarazadas, especialmente en las de clientela hospitalaria de caridad, pues es sabido que en nuestro medio la dieta aiimenticia no está bien balanceada, existiendo un déficit a favor de las harinas y en contra de las proteinas, grasas, sales minerales y vitaminas. Aun con esta anomalía de la alimentación, se observa con gran frecuencia en las embarazadas una hipoglicemia moderada, lo mismo que glicosurias pasajeras; estas glicosurias existiendo hipogiicemia, solamente son explicables por una baja en el umbral renal para la eliminación por la orina de la glucosa.

La lactacidemia en cambio está aumentada. Este aumento se ha querido explicar por un aumento de la desintegración glucogénica en los múscuios de la embarazada; también se cree sea debida a causas endocrinas, entre ellas la hiperfunción del tiroides y la insuficiencia en la función córtico-suprarrenal.

El glucógeno hepático se encuentra disminuído. Ya se anotó que existe un aumento en la ingestión de hidratos de carbono a la vez que un estado de hipoglicemia y disminución del glucógeno hepático. El aumento de oxidaciones durante ei embarazo podr.a ser la causa de estos fenómenos y el aumento en los requerimientos de glúcidos por parte del organismo fetal.

En cuanto al metabolismo de los prótidos se sabe que la embarazada retiene nitrógeno en su organismo.

Para algunos autores como Eufinger, la cantidad total de protetinas coioidales se encuentran disminuída, mientras para otros, entre ellos Plass, las proteínas totales están aumentadas; pero hay acuerdo en afirmar que las globulinas, especialmente el fibrinógeno, aparecen aumentadas. Esto tiene influencia sobre la oncosis del plasma.

La urea se encuentra disminuida; en cambio el ácido úrico se halla aumentado. Existe creatinuria en el embarazo, io mismo que aumento del amoníaco en la orina. 
La concentración de aminoácidos en ia sangre es normal.

La lipemia se encuentra aumentada; lo mismo sucede con la cetonemia. Esto se ha atribuido a las alteraciones deì hígado y de las glándulas endocrinas de la embarazada. También se atribuye al mayor consumo y aumento en las oxidaciones de los hidratos de carbono.

La colesterinemia también se encuentra aumentada. En la orina aparece aumentada la eliminación de ácidos grasos y especialmente, de cuerpos cetónicos, lo cual puede corregirse con una dieta rica en hidratos de carbono.

\section{Metabolismo de las sales minerales más importantes.}

Según varias investigaciones de diversos autores, entre ellos Timpe y Hellmuth, la calcemia no está fundamentalmente alteracla en ei embarazo. El calcio ionizado que es el que verdaderamente no sufre modificaciones, verificándose las modificaciones en la cifra total de calcio, a expensas de la fracción no ionizada y del que está en combinación orgánica.

El sodio en la sangre se encuentra disminuido y el cloro aumentado. En cambio en los tejidos sucede lo contrario con estos dos elementos, lo cual explica en parte ia tendencia a formar edemás en embarazos aun normales. El potasio según investigaciones recientes no experimenta cambios apreciables en embarazos normales, pero aumenta su concentración en la sangre en las gestosis.

También ha comprobado su retención en los tejidos de la gestante.

El hierro plasmático está elevado según Aibers (1941). Las necesidades maternas de hierro están aumentadas y el hierro de los depósitos maternos, disminuído.

En cuanto al yodo, los requerimientos de este elemento, en la gestación, lógicamente deben estar aumentados, por una parte, por el hiperfuncionamiento del tiroides, causa principal, y por otra parte debido a la demanda del feto de este elemento. La yodemia de las embarazadas está aumentada.

En los casos de hipertiroidismo en el embarazo, según un trabajo hecho a este respecto y publicado en "La Semana Médica Argentina" (1952), se saca en conclusión que el tratamiento con los antitiroideos es el único efectivo en los casos de enfermas hipertiroidianas en el embarazo. Antes del empleo de estos compuestos las pacientes embarazadas que sufrían de esta afección, no 
solo corrían los riesgos eventuales de su enfermedad, sino que había frecuentes abortos, y una elevada mortalidad fetal.

\section{Metabolismo del agua.}

Se sabe que en el embarazo como ya se anotó anteriormente, hay tendencia a la producción de edemas que se traducen en aumento de peso.

Las causas más frecuentes citadas de este fenómeno son: disminución de la presión encótica, retención de sodio y potasio $\in \mathrm{n}$ los tejidos, aumento de la presión venosa. Además en la retención hídrica de los tejidos, juegan papel importante, las hormonas retrohipofisiarias y los estrógenos (Anselmino y Hoffmann).

\section{Glándula tiroides y embarazo.}

Al hacer un examen cuidadoso del estado del tiroides en las pacientes estudiadas, comprobamos que en veintitrés de ellas existían grados variables de hipertrofia de esta glándula y en las veintisiete restantes, permanecieron normaies, clínicamente. Para tratar de explicar este fenómeno será bueno recordar los principales puntos referentes a la fisiología tiroidiana.

Se sabe que la hormona tiroidea, secreción interna de los folículos tiroideos, ejerce una extensa acción estimuiante sobre las oxidaciones en todo el organismo. Influye por lo tanto sobre el metabolismo de ios glúcidos, prótidos, lípidos, sales minerales y agua y vitaminas. Una de las funciones más importantes del tiroides es la relacionada con el metabolismo del yodo. El único yodo utilizado por el organismo es el que se emplea en la elaboración de la secreción tiroidiana. Ei aporte de yodo al organismo se hace por medio de la alimentación, en forma natural. En las regiones montañosas el aporte de este elemento es mucho menor que en las regiones marítimas, lo cual se traduce en una hiperplasia compensadora de la giándula. Samnson Wright, dice: "La tiroides aumenta de tamaño en el valiente pero infructuoso esfuerzo de fabricar tiroxina sin yodo".

Ahora bien: si tenemos en cuenta que el metabolismo basal en el embarazo se encuentra aumentado, como lo hemos demostrado, y por consiguiente todas las oxidaciones orgánicas son mayores, en este estado fisiológico los requerimientos de yodo serán mayores, y al no suministrar este elemento en la dieta alimenti- 
cia, existirá indefectiblemente una tendencia a la hipertrofia del tiroides en la gestante.

De 10 expuesto anteriormente podemos deducir que especialmente en las regiones montañosas, como son la mayor parte de las habitadas en nuestro país, en donde los alimentos son más pobres en yodo, sería muy conveniente la divulgación y el empieo de la sal yodada en forma discriminada, especialmente en los servicios de prenatal. En estos servicios también es necesario, en los casos en que se sospechen insuficiencias tiroideas, ya sea por hipertiroidismo o por hipotiroidismo, establecer tratamientos adecuados, con controles del metabolismo basal durante ei embarazo. Es indispensable también estabiecer una dieta bien balanceada, pues la mayoría de nuestro pueblo consume un exceso de hidrocarbonados, en tanto que las proteínas, las grasas, sales minerales y vitaminas, son muy reducidas o faltan en su ración diaria.

\section{RESUMEN Y COMENTARIOS}

Se practicó el examen de metabolismo basal a cincuenta embarazadas normales a término hospitalizadas en el Instituto Materno-Infantil "Concepción Viliaveces de Acosta".

De este examen pudimos comprobar que la mayor frecuencia de los valores del metabolismo basal en el embarazo normal a término corresponden a una cifra comprendida entre $+1 \mathrm{y}+$ $20 \%$.

Se encontraron también casos de $+50 \%$ y hasta $70 \%$ sin que hubiera anormalidad clínica manifiesta. Lo mismo podemos decir de algunos casos en que se encontró disminuído, siendo esta disminución hasta de $20 \%$.

Se encontró que en 23 de las 50 pacientes examinadas existia hipertrofia del tiroides de grado variable, io cual corresponde a un $46 \%$ del total de observaciones, cifra bastante alta que nos indica la tendencia que hay en el embarazo a producirse hipertrofias de esta glándula por deficiencia de yodo en la dieta alimenticia.

La principal causa del aumento del metabolismo basal en ei embarazo parece que corresponde a un hiperfuncionamiento del tiroides.

Las demás glándulas de secreción interna, a excepción del ovario, también influyen de manera coordinada sobre el aumento del metabolismo basal en el embarazo. En los casos de hipertiroidismo en ei embarazo, según estudios recientes, se saca en con- 
clusión que el único tratamiento racional y efectivo es el de los antitiroideos, evitando en esta forma los riesgos eventuales de la enfermedad y el peligro de abortos y de una elevada morbilidad fetal.

En el embarazo, aun en casos normales, hay tendencia a la formación de edemas, ios cuales se expiican por disminución de la presión oncótica del piasma, aumento de la presión venosa, retención de sodio y potasio en los tejidos.

También se cree que juegan papel importante en la retención hídrica de los tejidos las hormonas retrohipofisiarias y los estrógenos.

\section{BIBLIOGRAFIA}

1. SAMNSON WRIGHT.-“"Fisiología Aplicada". IV Edic.: 559-562. Edit. Nacional México. 1949.

2. HOUSSAY BERNARDO A.- “Fisiología Humana". II Edic. 471-493. Edit. El Ateneo. Buenos Aires. 1951.

3. DUQUE GOMEZ GONZALO._- "Metabolismo basal en el embarazo". Tesis de grado de la Facultad de Medicina (Universidad Nacional). Bogotá. 1947.

4. NUBIOLA-ZARATE._-“Tratado de Obstetricia”. 193-194 y 255-266. Ed't. labor. Barcelona. 1951.

5. L. SAAVEDRA M.- "Observaciones sobre Metabolismo basal en el embarazo". Tesis de grado de la Faccltad de Medicina (Universidad Nacional). 1954.

6. “La Semana Médica. Argentina, T. 100, número 9. Comentario editorial. 267, febrero 28 de 1952.

7. QUINTERO GOMEZ._- "Profilaxia y tratamiento del bocio simple". "Revista Médica". 3: 104-126. Mayo de 1945.

8. SMITH Y SMITH._- "Am. Journal of Obst. and Cyn". 39: 405. 1940

BOTELLA Y P. CASANOVA.- "Medicina". 2: 14-79 y 334 Edit. Labor. Barcelona. 1945. 